\title{
Immersion Meta-Lenses at Visible Wavelengths for Nanoscale Imaging
}

\section{Citation}

Chen, Wei Ting, Alexander Y. Zhu, Mohammadreza Khorasaninejad, Zhujun Shi, Vyshakh Sanjeev, and Federico Capasso. 2017. "Immersion Meta-Lenses at Visible Wavelengths for Nanoscale Imaging." Nano Letters 17 (5): 3188-94. https://doi.org/10.1021/acs.nanolett.7b00717.

\section{Permanent link}

http://nrs.harvard.edu/urn-3:HUL.InstRepos:41371327

\section{Terms of Use}

This article was downloaded from Harvard University's DASH repository, WARNING: This file should NOT have been available for downloading from Harvard University's DASH repository.

\section{Share Your Story}

The Harvard community has made this article openly available.

Please share how this access benefits you. Submit a story.

\section{Accessibility}




\title{
Immersion Meta-Lenses at Visible Wavelengths for Nanoscale Imaging
}

\author{
Wei Ting Chen, ${ }^{\dagger}$ Alexander Y. Zhu, ${ }^{\dagger}$ Mohammadreza Khorasaninejad, ${ }^{\dagger}$ Z Zhujun Shi, \\ Vyshakh Sanjeev, ${ }^{\dagger, \S}$ and Federico Capasso ${ }^{*}{ }^{\dagger}$ \\ ${ }^{\dagger}$ Harvard John A. Paulson School of Engineering and Applied Sciences and ${ }^{\ddagger}$ Department of Physics, Harvard University, Cambridge, \\ Massachusetts 02138, United States \\ ${ }^{\S}$ University of Waterloo, Waterloo, Ontario N2L 3G1, Canada
}

Supporting Information

ABSTRACT: Immersion objectives can focus light into a spot smaller than what is achievable in free space, thereby enhancing the spatial resolution for various applications such as microscopy, spectroscopy, and lithography. Despite the availability of advanced lens polishing techniques, hand-polishing is still required to manufacture the front lens of a high-end immersion objective, which poses major constraints for lens design. This limits the shape of the front lens to spherical. Therefore, several other lenses need to be cascaded to correct for spherical aberration, resulting in significant challenges for miniaturization and adding design complexity for different immersion liquids. Here, by using metasurfaces, we

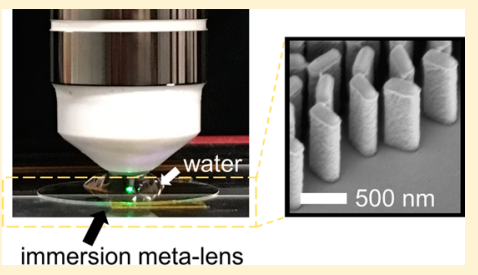
demonstrate liquid immersion meta-lenses free of spherical aberration at various design wavelengths in the visible spectrum. We report water and oil immersion meta-lenses of various numerical apertures (NA) up to 1.1 and show that their measured focal spot sizes are diffraction-limited with Strehl ratios of approximately 0.9 at $532 \mathrm{~nm}$. By integrating the oil immersion meta-lens $(\mathrm{NA}=1.1)$ into a commercial scanning confocal microscope, we achieve an imaging spatial resolution of approximately $200 \mathrm{~nm}$. These meta-lenses can be easily adapted to focus light through multilayers of different refractive indices and mass-produced using modern industrial manufacturing or nanoimprint techniques, leading to cost-effective high-end optics.

KEYWORDS: Metasurface, immersion meta-lens, visible spectrum, titanium dioxide, high numerical aperture

$\mathrm{T}$ he immersion technique is widely used to enhance spatial resolution of a lithography or imaging system by adding a layer of liquid between the front lens of an objective and the specimen. One example is the use of water immersion lenses in deep ultraviolet steppers in semiconductor manufacturing. ${ }^{1}$ This enables the fabrication of complementary metal-oxidesemiconductor (CMOS) gates with widths of a few tens of nanometers using excimer lasers at a $193 \mathrm{~nm}$ wavelength. In microscopy, the crucial component of an high-end immersion objective is the front lens: its shape is usually plano-convex with a diameter of a few millimeters. The convex surface possesses a large curvature to provide sufficient optical power (the reciprocal of the focal length of a lens). ${ }^{2}$ This makes its fabrication very challenging and requires hand-polishing. ${ }^{3}$ Additional lenses also need to be cascaded to reduce the spherical aberration induced by the spherical shape of the front lens, increasing the overall device volume, design complexity, and cost. These factors pose a significant challenge to the mass production, miniaturization, and customization of immersion lenses in general.

In recent years, metasurfaces consisting of subwavelength structures patterned on a substrate have attracted increasing attention due to their ability to simultaneously control the amplitude, phase, and polarization of light in a compact configuration. ${ }^{4-6}$ The thickness of a metasurface (excluding its supporting substrate) is on the scale of a wavelength, which provides a platform with which to realize compact optical devices, such as holograms, ${ }^{7-9}$ polarimeters, ${ }^{10-12}$ modulators, $^{13,14}$ and lenses. ${ }^{15-20}$ These devices can be realized with high efficiency by using plasmonic ${ }^{21}$ or high refractive index dielectric nanostructures ${ }^{22-24}$ in reflection and transmission configurations, respectively. Here, by utilizing $\mathrm{TiO}_{2}$ nanofins fabricated with atomic layer deposition $(\mathrm{ALD})$, we report the first planar water and oil immersion lenses (referred to as metalenses herein) with numerical apertures (NA) up to 1.1 in the visible. Note that our immersion meta-lenses are designed using subwavelength nanostructures, which provides more-precise and -efficient phase control compared to binary amplitude and phase Fresnel zone plates. ${ }^{25,26}$ Our water immersion metalenses show diffraction-limited focal spots with Strehl ratios higher than 0.9 at their design wavelength of $\lambda_{\mathrm{d}}=532 \mathrm{~nm}$. These meta-lenses can be tailored for any immersion liquid. As an example, we also show oil immersion meta-lenses with diffraction-limited focal spots, at $\lambda_{\mathrm{d}}=532 \mathrm{~nm}$ and $\lambda_{\mathrm{d}}=405 \mathrm{~nm}$, with Strehl ratios higher than 0.8. By integrating the meta-lens designed at $\lambda_{\mathrm{d}}=532 \mathrm{~nm}$ with a commercial scanning confocal microscope, we achieve an imaging spatial resolution as small as $200 \mathrm{~nm}$.

Received: February 17, 2017

Revised: April 5, 2017

Published: April 7, 2017 
(a)

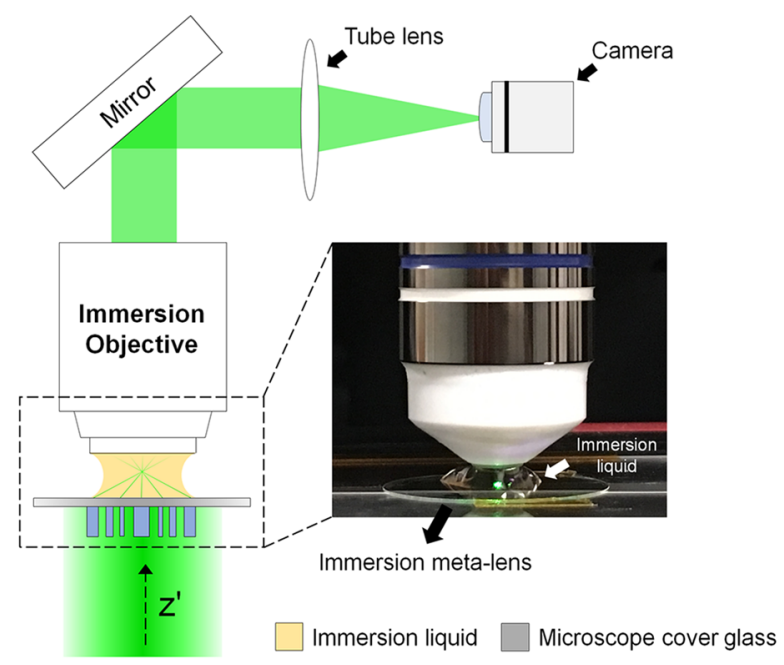

(b)

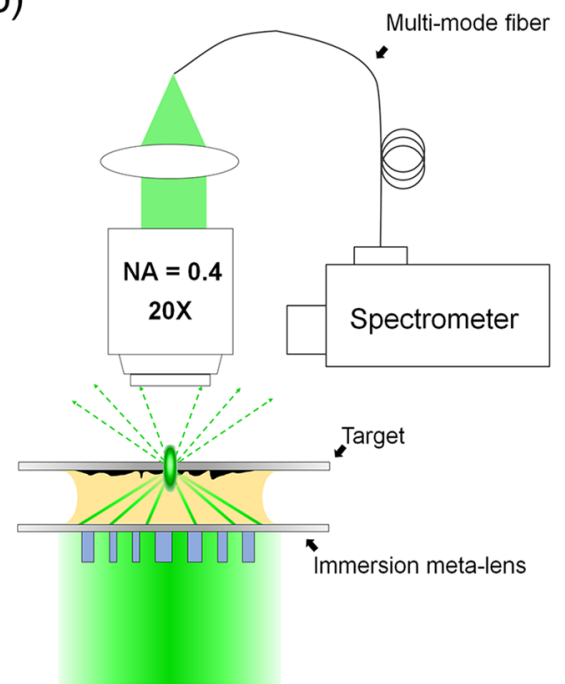

Figure 1. (a) Schematic measurement setup for characterizing the meta-lenses. The inset shows an image taken when measuring a water immersion meta-lens with NA $=0.9$ at $532 \mathrm{~nm}$. A relative coordinate $\left(x^{\prime}, y^{\prime}, z^{\prime}\right)$ is defined with its origin at the focus. The green spot results from the scattering of focused light in water. (b) Schematic for a commercial scanning confocal microscope integrated with an oil immersion meta-lens. The oil immersion meta-lens focuses normally incident light to a diffraction-limited spot on a target. The target was fabricated on a gold-coated cover glass substrate, and was scanned by moving a piezo stage. The scattered light was collected by a Nikon objective designed for imaging through a cover glass with a thickness of $170 \mu \mathrm{m}$.

(a)

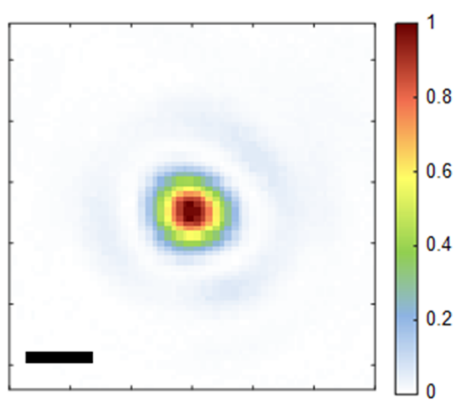

(d)

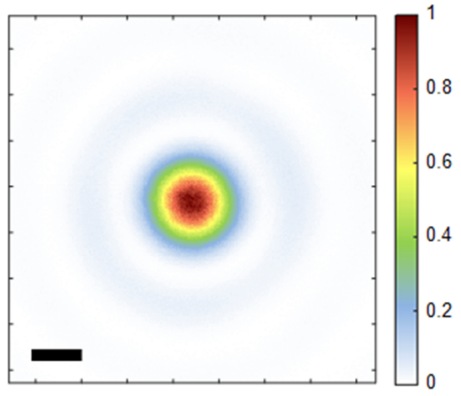

(b)

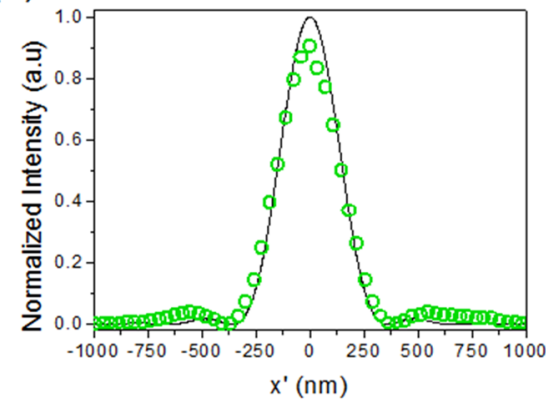

(e)

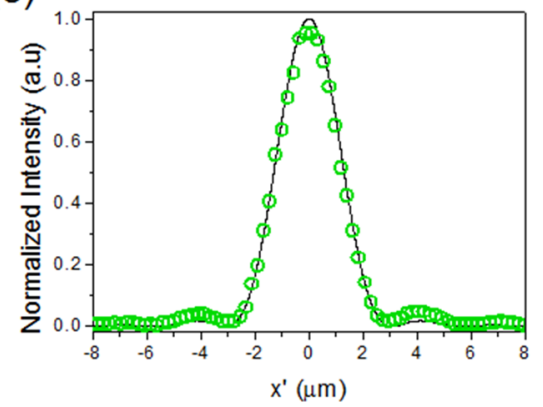

(c)

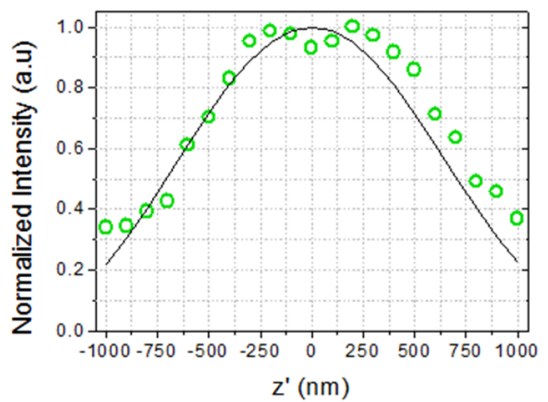

(f)

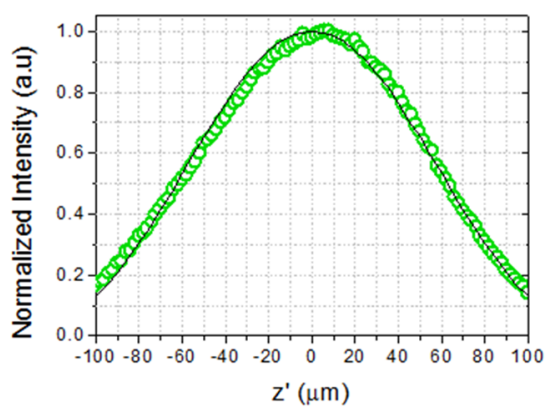

Figure 2. Focusing characterization of water immersion meta-lenses at the design wavelength $\lambda_{\mathrm{d}}=532 \mathrm{~nm}$. (a) Normalized intensity profile of the focal spot from the meta-lens with NA $=0.9$. Scale bar: $400 \mathrm{~nm}$. (b) The horizontal cut of panel (a) with its intensity normalized to its corresponding diffraction-limited Airy disk (black curve) for a same given area. The Strehl ratio can be obtained by dividing the peak intensity value of measured (green dots) by that expected from theory (black curve). (c) Measured intensity variation at the center of the focal spot (green dots) along propagation direction $\left(z^{\prime}\right.$ axis shown in Figure 1). Black curve shows theoretical prediction from OpticsStudio (Zemax Inc.). The depth of focus can be estimated from the width of the curve at a normalized intensity equals to 0.8 . $(\mathrm{d}-\mathrm{f})$ Corresponding analysis for a meta-lens with NA $=0.1$. Scale bar in panel (d): $2 \mu \mathrm{m}$.

Results. Design and Fabrication of Immersion MetaLenses. Our immersion meta-lenses are designed in an infiniteconjugate configuration. A collimated plane-wave sequentially passes through the nanofins, which impart a given phase profile $\varphi(x, y)$, and a microscope cover glass before being focused in an immersion liquid. Note that in this configuration, the nanostructures are not directly in contact with liquid. This not only provides protection when the immersion meta-lenses are used in imaging but also prevents the lowering of efficiency due to reduction of the refractive index contrast between $\mathrm{TiO}_{2}$ 
(a)

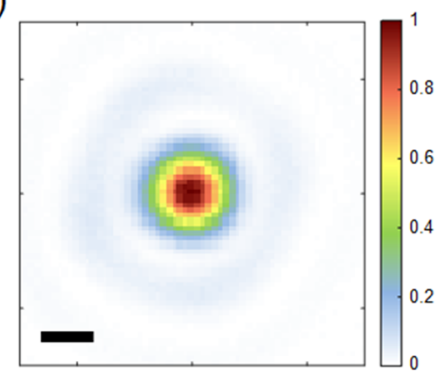

(d)

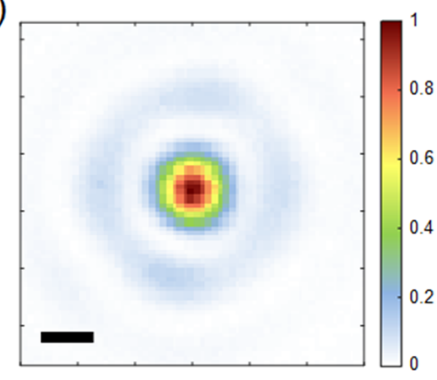

(b)

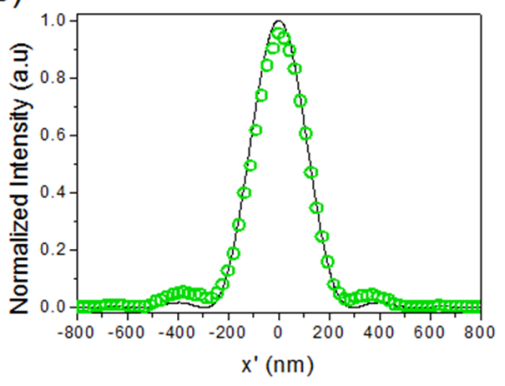

(e)

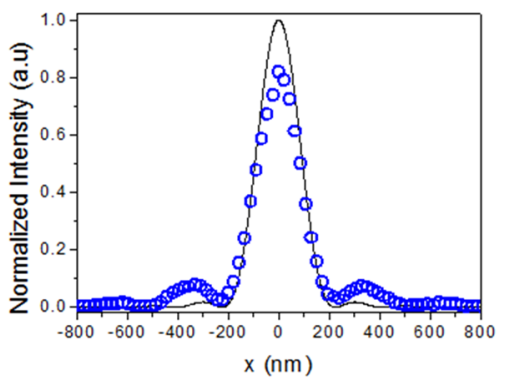

(c)

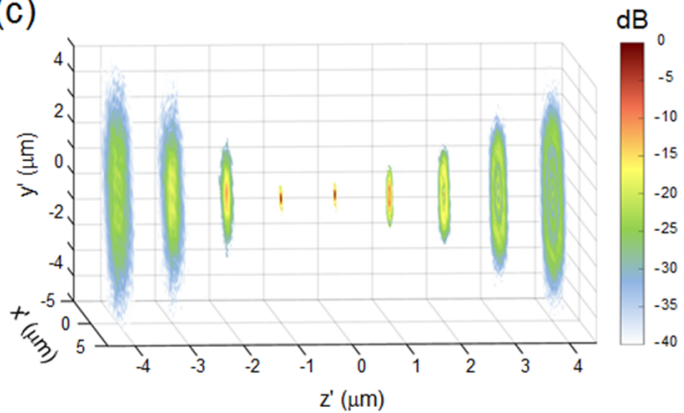

(f)

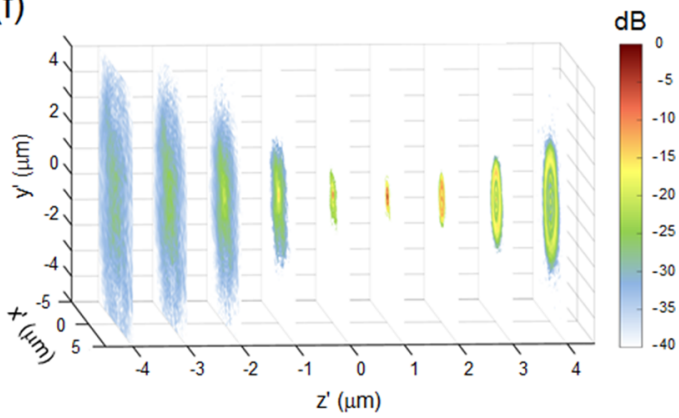

Figure 3. Focusing characterization of oil immersion meta-lenses with NA $=1.1$ at their design wavelengths. (a) Normalized intensity profile of a focal spot from the meta-lens designed at $532 \mathrm{~nm}$. (b) Intensity distribution (green dots) from the horizontal cut of (a) normalized to the intensity of a diffraction-limited Airy disk (black curve) for a given area. (c) Intensity distribution in $\mathrm{dB}$ scale on the $x^{\prime}-y^{\prime}$ plane, showing the evolution of the beam from $4 \mu \mathrm{m}$ before to $4 \mu \mathrm{m}$ after the focus. (d-f) Corresponding analysis of panels a-c for a meta-lens designed at $405 \mathrm{~nm}$. Scale bar for panels a and $\mathrm{d}$ is $200 \mathrm{~nm}$.

nanofins and their surrounding medium. We designed immersion meta-lenses for two different liquids: water and oil. The refractive index of the oil is chosen to approximately match that of the cover glass substrate (Figure S1). The phase profile $\varphi(x, y)$ at design wavelength $\lambda_{\mathrm{d}}$ for a given position $(x, y)$ can be obtained using the ray-tracing method such that all rays arrive at the focal spot in phase. Here, we use a commercial software (OpticsStudio, Zemax LLC) to determine the optimal phase profile $\varphi(x, y)$ (see the Methods section). By utilizing the geometric phase principle, the desired $\varphi(x, y)$ was subsequently imparted for left-handed circularly polarized incident light by the rotation of each nanofin at $(x, y)$ through the relation $\varphi(x, y)=2 \alpha$, where $\alpha$ is the rotation angle of a nanofin. The unit cell size $p$, width $w$, length $l$, and height $h$ of an individual nanofin are optimized by parameter sweep using threedimensional finite difference time domain (FDTD) method (Lumerical Inc.) to maximize polarization conversion efficiency at the design wavelength $\lambda_{\mathrm{d}}$ (see the Methods section). The maximum polarization conversion efficiency is achieved when the nanofins function as half-wave plates. The parameters $(w, l$, $h$, and $p$ ) for meta-lenses designed at $\lambda_{\mathrm{d}}=532 \mathrm{~nm}$ and $\lambda_{\mathrm{d}}=405$ $\mathrm{nm}$ are $80,220,600$, and $240 \mathrm{~nm}$ and $60,120,600$, and 150 $\mathrm{nm}$, respectively. The immersion meta-lenses were fabricated with the approach described in ref 8 . The use of ALD in our fabrication process not only ensures low surface roughness but also straight sidewalls compared to dry-etching process. ${ }^{27}$ The scanning electron microscope images are provided in Figure S2.

It is worth noting that the phase profile $\varphi(x, y)$ is discretely imparted due to the finite unit cell size $p$ in the design of the nanofins, which in turn limits the maximum achievable NA. This can be understood by the Nyquist-Shannon sampling theorem in the spatial domain. The maximum transverse wavenumber provided by a meta-lens at $\lambda_{\mathrm{d}}$ is $k_{\max }=\frac{1}{\lambda_{\mathrm{d}}} \cdot \mathrm{N} A$, where NA is the designed numerical aperture at $\lambda_{\mathrm{d}}$. To prevent spherical aberration, the condition must then be satisfied:

$$
p \leq \frac{\lambda_{\mathrm{d}}}{2 \cdot N A}
$$

For example, in our case, the meta-lens designed at $\lambda_{\mathrm{d}}=532$ $\mathrm{nm}$ and $\lambda_{\mathrm{d}}=405 \mathrm{~nm}$ have $p=240 \mathrm{~nm}$ and $p=150 \mathrm{~nm}$, respectively. This corresponds to a maximum achievable NA of 1.1 and 1.35 , respectively. The smaller the $p$, the larger the achievable NA, and, consequently, the higher the efficiency of the meta-lens due to better sampling. However, for a given set of design parameters ( $w, l$, and $h)$, the peak polarization conversion efficiency of the nanofin blue-shifts as $p$ decreases. To maintain the maximal efficiency at $\lambda_{\mathrm{d}}$, one needs to either increase the ratio of $l$ to $w$ or the height $h$, which is ultimately limited by fabrication constrains.

Characterization of Immersion Meta-Lenses. Figure $1 \mathrm{a}, \mathrm{b}$ shows the schematic setup for characterizing the immersion meta-lenses and the setup used for nanoscale imaging, respectively (see the Methods section). We define the relative $z^{\prime}$ axis with its origin at the center of focal spot in Figure 1a for convenience. Because the focal spots of meta-lenses are embedded inside immersion liquids, to minimize the aberrations of the measurement system, water or oil immersion objectives with NAs higher than that of immersion meta-lenses were selectively used. Figure 2 shows the characterization of focal spots for the water immersion meta-lenses with $\mathrm{NA}=0.9$ (first row) and NA $=0.108$ (second row, referred to as $\mathrm{NA}=$ 0.1 hereafter for simplicity) designed at $\lambda_{\mathrm{d}}=532 \mathrm{~nm}$. Figure $2 \mathrm{a}$ shows a highly symmetric focal spot with an average full-width at half-maximum (fwhm) of $316 \pm 13 \mathrm{~nm}$ and a Strehl ratio of 0.9 (Figure $2 \mathrm{~b}$ ). This demonstrates that the water immersion meta-lens meets the requirement for diffraction-limited focusing: ${ }^{28}$ fwhm $\approx 0.51 \lambda / \mathrm{NA}$ and Strehl ratio $\geq 0.8$. The 
(a)

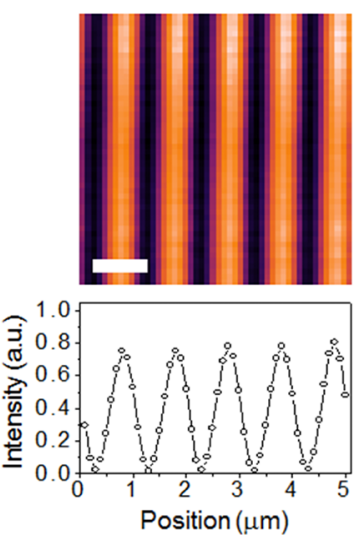

(d)

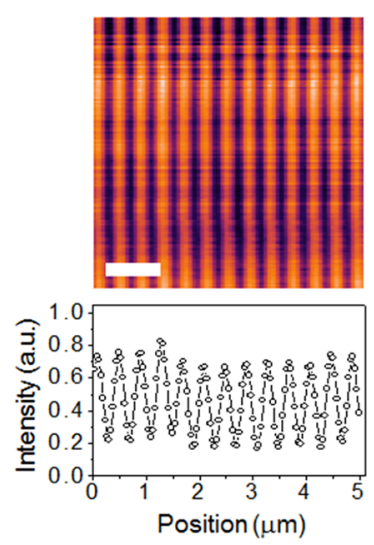

(b)
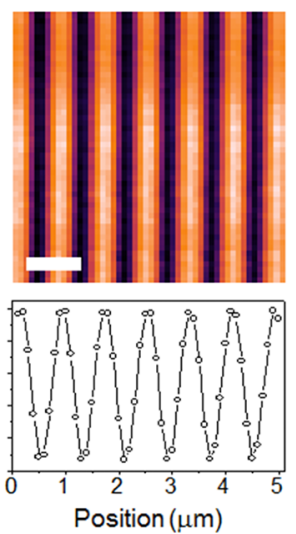

(e)

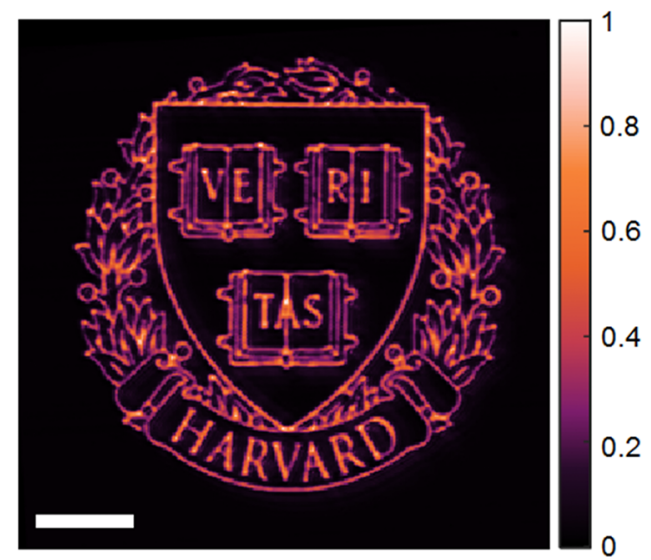

(c)

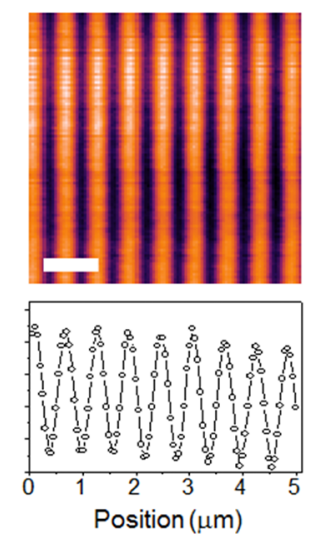

0.4

0.2

Figure 4. Confocal imaging with oil immersion meta-lens design at wavelength $\lambda=532 \mathrm{~nm}$ with NA $=1.1$. (a-d) Scanning images for metallic stripes fabricated by e-beam lithography followed by metal deposition and lift-off process. Scale bar: $1 \mu \mathrm{m}$. The insets show mean peak-to-peak values of (a) $1 \mathrm{um},(\mathrm{b}) 783 \mathrm{~nm}$, (c) $593 \mathrm{~nm}$, and (d) $400 \mathrm{~nm}$ with standard deviations smaller than $10 \%$. The piezo was moved by 100, 50 , and $37.5 \mathrm{~nm}$ per step for panels a and b, panel c, and panel d, respectively. (e) Scanning image of a Harvard logo for a larger scanning region of $60 \mu \mathrm{m} \times 60 \mu \mathrm{m}$. The piezo was moved by $200 \mathrm{~nm}$ per step. This target was fabricated by focused ion beam milling. Scale bar: $10 \mu \mathrm{m}$.

focusing efficiency of this meta-lens is wavelength-dependent and peaks at $42 \%$ at wavelength $\lambda=550 \mathrm{~nm}$ (Figure S3). The focusing efficiency is defined as the power of focal spot divided by incident light in the case of circularly polarized light. To measure the depth of focus (DOF), the water immersion objective was moved step by step vertically using a stepper motor; an image was recorded for each step corresponding to different $z^{\prime}$ planes shown in Figure 1a. This process maps the intensity distribution of the focal spot (Figure S4). In Figure 2c, we subsequently plot the intensity (green dots) at the center of focal spot along the $z^{\prime}$ direction (optical axis) normalized to the maximum intensity in the focal region, while the black curve shows the numerical prediction from OpticsStudio (Zemax LLC). The measured DOF corresponds to the difference of right and left boundary for the region with normalized intensity larger than 0.8. The theoretical DOF can be deduced using the optical analogue of the uncertainty principle ${ }^{29}$ given by

$$
\mathrm{DOF}=\frac{\lambda}{2 n[1-\cos (\theta)]}
$$

where $n$ is the refractive index of immersion liquid, $\theta=\sin ^{-1}\left(\frac{\mathrm{NA}}{n}\right)$ is the maximum diffraction angle at the edge of metalens. Note that eq 2 becomes $\frac{n \lambda}{\mathrm{NA}^{2}}$ for small NAs, giving the well-known approximation that the DOF is inversely proportional to the square of NA. Similar analysis for a lower $\mathrm{NA}=0.1$ water immersion meta-lens is shown in Figure $2 \mathrm{~d}-\mathrm{f}$; the averaged fwhm, Strehl ratio, and DOF are $2.51 \pm 0.02 \mu \mathrm{m}$, 0.97 , and $70 \mu \mathrm{m}$, respectively. For low NA, the experimental data agrees better with the results from OpticsStudio because its DOF is larger, implying larger vibration tolerance in measurement.

Figure 3a shows an image of focal spot for the oil immersion meta-lens with NA $=1.1$ designed at wavelength $\lambda_{\mathrm{d}}=532 \mathrm{~nm}$. It has a focal spot with average fwhm $=240 \pm 4 \mathrm{~nm}$ and a Strehl ratio of 0.94 (Figure $3 b$ ). Figure $3 c$ shows the focal spot intensity profile of this meta-lens in different $x^{\prime}-y^{\prime}$ planes. The negligible background signal demonstrates excellent phase realization, where the beam converges to a diffraction-limited spot. Our immersion meta-lens can also be designed at any wavelength in the visible. Figure $3 \mathrm{~d}-\mathrm{f}$ shows similar characterization using an oil immersion meta-lens designed at $405 \mathrm{~nm}$ : the focal spot (Figure 3d), average fwhm and Strehl ratio (203 $\pm 3.5 \mathrm{~nm}$ and 0.82 , respectively; Figure $3 \mathrm{e}$ ), and intensity versus the background (Figure 3f). The peak focusing efficiencies of these meta-lenses are 53\% and 32\% (Figure S5). The focusing efficiency and Strehl ratio for the meta-lens designed at $405 \mathrm{~nm}$ are lower compared to its counterpart at $532 \mathrm{~nm}$ because its constituent structure size is smaller, which results in a lower tolerance for fabrication errors. 
Immersion Meta-Lens for Diffraction-Limited Imaging. Our immersion meta-lens is designed for normal incidence at a given wavelength, implying it only corrects all monochromatic aberrations for an on-axis point source. If one uses these metalenses for wide-field imaging, due to the spatial extent of the object, monochromatic aberrations (especially coma) will reduce the spatial resolution. To overcome this limitation and achieve diffraction-limited imaging over a larger area, one can perform scanning imaging instead of wide-field imaging. Therefore, we integrate our oil immersion meta-lens into a commercial scanning confocal microscope (Witek, Alpha 300RS); see Figure 1b. The target was mounted on a piezo stage and scanned horizontally by a diffraction-limited focal spot from an oil immersion meta-lens with NA $=1.1$ designed at $\lambda_{\mathrm{d}}=532 \mathrm{~nm}$. The scattered light was collected by an achromatic refractive objective (Nikon achromat, CFI LWD, $\mathrm{NA}=0.4,20 \times)$ and subsequently focused into a multimode fiber with a core diameter of $50 \mu \mathrm{m}$ connected to a spectrometer and its associated charge-coupled device (CCD) camera. For each movement of the piezo stage, a spectrum was recorded, and the CCD counts at $532 \mathrm{~nm}$ were taken to contribute to the intensity of a pixel shown in Figure $4 a-e$. Such a configuration is also able to obtain photoluminescence or Raman images. Figure $4 \mathrm{a}-\mathrm{d}$ shows the images of resolution targets: metallic stripes designed with equal line widths and gaps of 500, 400, 300, and $200 \mathrm{~nm}$, respectively. The insets show the intensity along the horizontal direction through the center of each image. Figure $4 \mathrm{~d}$ is a slightly blurred and the intensity contrast is lower because the feature size approaches the resolution limit of the confocal microscope. ${ }^{30}$ Figure $4 \mathrm{e}$ shows an image with a larger scanning area for a Harvard logo (SEM images of the Harvard logo are provided in Figure S6).

Discussion. Our immersion meta-lenses are designed by the geometric phase principle, which can only focus and collect light for a specific circular polarization. For the applications that require polarization insensitive meta-lenses, we can use waveguiding effects to impart the required phase. For example, one can use nanopillars with circular cross-sections and control the phase by changing their diameters. ${ }^{31}$ The proposed immersion meta-lenses are also monochromatic. Its operation bandwidth can be expanded by engineering the resonance or dispersion of nanostructures, ${ }^{32-34}$ increasing the height of nanostructures to cover phase modulation for more than $2 \pi$ radians (multiorder or harmonic diffractive lenses) ${ }^{35,36}$ or adding a refractive lens to the meta-lens because they have opposite chromatic dispersions. ${ }^{37} \mathrm{We}$ also note that the socalled superoscillatory and supercritical lenses have been shown to be capable of focusing light in far-field with focal spot sizes that are smaller than the diffraction-limit. ${ }^{38-40}$ However, the efficiencies of these lenses are still yet to be improved, and the focal spots are accompanied by strong side-lobes, which are severe downsides in applications such as photoluminescence and nonlinear scanning imaging.

We have demonstrated scanning microscopic imaging through stage scanning, which is slower compared to the use of galvo mirror for laser scanning microscopy. The galvo mirror usually consists of a pair of small mirrors to rapidly deflect the laser beam. High-speed scanning microscopy can be implemented using meta-lens by adding another layer of metasurface to correct the aberrations (mainly coma aberration) such that the focal spot is still diffraction-limited for oblique incidence, ${ }^{41}$ or by integrating an array of immersion meta-lenses to reduce the scanning area of each meta-lens. The latter is promising for achieving the large field of view $(\sim \mathrm{cm} \times$ $\sim \mathrm{cm}$ ) required in many applications, especially in laser lithography, where the accuracy of a galvo mirror is insufficient. $^{42-44}$

Cover glasses usually have $\sim \pm 5 \mu \mathrm{m}$ error in thickness. In case of designing water immersion meta-lens, the inaccuracy of substrate thickness can induce spherical aberration. This results in focal length shift and might broaden the focal spot if the spherical aberration is larger than the tolerance of meta-lens, which is dependent on the NA; the smaller the NA, the larger the tolerance. In Figure S7, we show that the water immersion meta-lens can still be diffraction-limited up to $\mathrm{NA}=1.1$ considering the $\pm 5 \mu \mathrm{m}$ thickness error.

Note that our immersion meta-lenses can be tailored not just for any immersion liquid but also for multiple layers of different refractive indices. This is especially important for biorelated imaging. Conventional immersion objectives are designed for a single layer of immersion liquid; this introduces significant spherical aberrations when they are used to focus light into, e.g., biological tissue. ${ }^{45}$ Our immersion meta-lens can be designed by considering the refractive indices of epidermis and dermis to focus light in the tissue under human skin with no additional design and fabrication complexity.

Conclusions. This first demonstration of liquid immersion planar meta-lenses with NAs up to 1.1 conclusively shows that their versatility to be tailored for any liquid and are capable of providing diffraction-limited focal spots at their design wavelengths with focusing efficiencies about 50\%. By integrating the meta-lens with a commercial scanning confocal microscope, we achieved diffraction-limited imaging with spatial resolution of $\sim 200 \mathrm{~nm}$ at wavelength $\lambda=532 \mathrm{~nm}$. These metalenses can be designed taking into account the refractive index of multilayers, allowing numerous applications in optical lithography, laser-based microscopy and spectroscopy. The single-step lithographic fabrication of meta-lenses has the potential to overcome the drawbacks or challenges of lenspolishing techniques that have been used for centuries and has the possibility to be mass-produced with existing foundry technology (deep-UV steppers) or nanoimprinting for costeffective high-end immersion optics.

Methods. Phase Profile of Immersion Meta-Lenses. The phase profiles were calculated using a commercial ray-tracing software OpticsStudio from Zemax Inc., considering the configuration shown in Figure 1. The thickness of cover glass substrate was measured by a micrometer (CPM1, Thorlabs Inc.) and then input into OpticsStudio as a parameter. The phase profiles of immersion meta-lenses are described by a polynomial:

$$
\varphi(r)=\sum_{i=1}^{N} a_{i}\left(\frac{r}{R}\right)^{2 N}
$$

where $R$ is the radius of immersion meta-lens, and $r=\sqrt{x^{2}+y^{2}}$ is the polar coordinate. The coefficients $a_{i}$ were optimized through an algorithm in OpticsStudio for minimizing the spread of the cross-point of each ray on focal plane. See Supplementary Table S1 for the coefficients of all immersion meta-lenses.

Measurement Setup for Characterizing the Focal Spots. Meta-lenses were characterized using a custom-built microscope consisting of a fiber-coupled laser source, linear polarizer, quarter-waveplate, and an immersion objective lens paired with 
its tube lens to form an image on a CMOS camera with a pixel size of $2.2 \mu \mathrm{m}$. For measuring the water and oil immersion meta-lenses, the objective used was an Olympus water immersion objective (LUMPLFLN, 60×, NA = 1) and a Nikon oil immersion objective (CFI, $100 \times, \mathrm{NA}=1.25)$ paired with their corresponding tube lenses of focal length $f=180 \mathrm{~mm}$ and $200 \mathrm{~mm}$, respectively.

Simulation. Three-dimensional full wave simulation was performed by a commercial software (Lumerical Inc.) based on the FDTD. We arranged an array of $\mathrm{TiO}_{2}$ nanofins in such a way that it diffracts light with conversed polarization state to a particular angle. Periodic and perfectly matched layer boundary conditions were used along transverse and longitudinal directions with respect to the propagation of incident circularly polarized light. The length and width of the $\mathrm{TiO}_{2}$ nanofin were swept within a region considering fabrication limitations to maximize the polarization conversion efficiency. Polarization conversion efficiency is calculated by dividing the total diffracted optical power around the particular angle by the input optical power.

\section{ASSOCIATED CONTENT}

\section{S Supporting Information}

The Supporting Information is available free of charge on the ACS Publications website at DOI: 10.1021/acs.nanolett.7b00717.

Figures showing the refractive index of immersion oil and cover glass substrate, scanning electron microscope images, measured focusing efficiencies of oil and water immersion meta-lenses, focal spot profiles and focusing efficiency for water immersion meta-lenses, and simulated aberration analysis. A table showing polynomial coefficients of immersion meta-lens phase profiles. (PDF)

\section{AUTHOR INFORMATION}

\section{Corresponding Author}

*E-mail: capasso@seas.harvard.edu.

\section{ORCID 1}

Wei Ting Chen: 0000-0001-8665-9241

Mohammadreza Khorasaninejad: 0000-0002-0293-9113

\section{Author Contributions}

W.T.C. and A.Y.Z. contributed equally to this manuscript. W.T.C., M.K., and F.C. conceived of the study. W.T.C and M.K. performed the FDTD simulations. A.Y.Z. developed the process and fabricated the samples. W.T.C., A.Y.Z., and Z.S. characterized the meta-lenses. W.T.C. and V.S. analyzed the results. W.T.C., A.Y.Z., M.K., and F.C. co-wrote the paper. All authors discussed and commented on the manuscript.

\section{Notes}

The authors declare no competing financial interest.

\section{ACKNOWLEDGMENTS}

This work was supported in part by the Air Force Office of Scientific Research (MURI, grant nos. FA9550-14-1-0389 and FA9550-16-1-0156) and Thorlabs Inc. A.Y.Z. thanks Harvard SEAS and A*STAR Singapore under the National Science Scholarship scheme. R.C.D. is supported by a Charles Stark Draper Fellowship. This work was performed in part at the Center for Nanoscale Systems (CNS), a member of the National Nanotechnology Coordinated Infrastructure (NNCI), which is supported by the National Science Foundation under NSF award no. 1541959. CNS is a part of Harvard University.

\section{REFERENCES}

(1) Lin, B. J. J. Micro/Nanolithogr., MEMS, MOEMS 2004, 3, 377395.

(2) Kurvits, J. A.; Jiang, M.; Zia, R. J. Opt. Soc. Am. A 2015, 32 (11), 2082-2092.

(3) Nikon. Lens Polishing - Hand-polishing spherical front lenses for microscopes. https://www.nikoninstruments.com/Learn-Explore/ Nikon-Craftsmanship/Lens-Polishing-Hand-polishing-spherical-frontlenses-for-microscopes (accessed April 4, 2017).

(4) Lin, D.; Fan, P.; Hasman, E.; Brongersma, M. L. Science 2014, 345, 298-302.

(5) Yu, N.; Capasso, F. Nat. Mater. 2014, 13, 139-150.

(6) Kildishev, A. V.; Boltasseva, A.; Shalaev, V. M. Science 2013, 339, 1232009.

(7) Huang, Y. W.; Chen, W. T.; Tsai, W. Y.; Wu, P. C.; Wang, C. M.; Sun, G.; Tsai, D. P. Nano Lett. 2015, 15, 3122-3127.

(8) Devlin, R. C.; Khorasaninejad, M.; Chen, W. T.; Oh, J.; Capasso, F. Proc. Natl. Acad. Sci. U. S. A. 2016, 113, 10473-10478.

(9) Li, X.; Chen, L.; Li, Y.; Zhang, X.; Pu, M.; Zhao, Z.; Ma, X.; Wang, Y.; Hong, M.; Luo, X. Science Advances 2016, 2, e1601102.

(10) Chen, W. T.; Török, P.; Foreman, M.; Liao, C. Y.; Tsai, W.-Y.; Wu, P. R.; Tsai, D. P. Nanotechnology 2016, 27, 224002.

(11) Mueller, J. P. B.; Leosson, K.; Capasso, F. Optica 2016, 3, 4247.

(12) Pors, A.; Nielsen, M. G.; Bozhevolnyi, S. I. Optica 2015, 2, 716723.

(13) Zheludev, N. I. Science 2015, 348, 973-974.

(14) Engheta, N. Science 2007, 317, 1698-1702.

(15) Khorasaninejad, M.; Chen, W. T.; Zhu, A. Y.; Oh, J.; Devlin, R. C.; Rousso, D.; Capasso, F. Nano Lett. 2016, 16, 4594-4600.

(16) Arbabi, A.; Horie, Y.; Ball, A. J.; Bagheri, M.; Faraon, A. Nat. Commun. 2015, 6, 7069.

(17) Khorasaninejad, M.; Chen, W. T.; Devlin, R. C.; Oh, J.; Zhu, A. Y.; Capasso, F. Science 2016, 352, 1190-1194.

(18) Chen, W. T.; Khorasaninejad, M.; Zhu, A. Y.; Oh, J.; Devlin, R. C.; Zaidi, A.; Capasso, F. Light Sci. Appl. 2017, 6, e16259.

(19) Zentgraf, T.; Liu, Y.; Mikkelsen, M. H.; Valentine, J.; Zhang, X. Nat. Nanotechnol. 2011, 6, 151-155.

(20) Ho, J. S.; Qiu, B.; Tanabe, Y.; Yeh, A. J.; Fan, S.; Poon, A. S. Y. Phys. Rev. B: Condens. Matter Mater. Phys. 2015, 91, 125145.

(21) Sun, S.; Yang, K.-Y.; Wang, C.-M.; Juan, T.-K.; Chen, W. T.; Liao, C. Y.; He, Q.; Xiao, S.; Kung, W.-T.; Guo, G.-Y.; Zhou, L.; Tsai, D. P. Nano Lett. 2012, 12, 6223-6229.

(22) Arbabi, A.; Horie, Y.; Bagheri, M.; Faraon, A. Nat. Nanotechnol. 2015, 10, 937-943.

(23) Decker, M.; Staude, I.; Falkner, M.; Dominguez, J.; Neshev, D. N.; Brener, I.; Pertsch, T.; Kivshar, Y. S. Adv. Opt. Mater. 2015, 3, 813-820.

(24) Lu, F.; Sedgwick, F. G.; Karagodsky, V.; Chase, C.; ChangHasnain, C. J. Opt. Express 2010, 18, 12606-12614.

(25) Schonbrun, E.; Rinzler, C.; Crozier, K. B. Appl. Phys. Lett. 2008, 92, 071112 .

(26) Chao, D.; Patel, A.; Barwicz, T.; Smith, H. I.; Menon, R. J. Vac. Sci. Technol., B: Microelectron. Process. Phenom. 2005, 23, 2657-2661.

(27) Lalanne, P.; Astilean, S.; Chavel, P.; Cambril, E.; Launois, H. J. Opt. Soc. Am. A 1999, 16, 1143-1156.

(28) Thorlabs. Super Apochromatic Microscope Objectives. https:// www.thorlabs.com/newgrouppage9.cfm?objectgroup_id=9895 (accessed April 4, 2017).

(29) Mansuripur, M. Classical optics and its applications; Cambridge University Press: Cambridge, U.K., 2009; pp 258-273.

(30) Keller, H. E. Objective Lenses for Confocal Microscopy. In Handbook Of Biological Confocal Microscopy; Pawley, J. B., Ed.; Springer US: Boston, MA, 2006; pp 145-161. 
(31) Khorasaninejad, M.; Zhu, A. Y.; Roques-Carmes, C.; Chen, W. T.; Oh, J.; Mishra, I.; Devlin, R. C.; Capasso, F. Nano Lett. 2016, 16, 7229-7234.

(32) Li, Y.; Li, X.; Pu, M.; Zhao, Z.; Ma, X.; Wang, Y.; Luo, X. Sci. Rep. 2016, 6, 19885.

(33) Aieta, F.; Kats, M. A.; Genevet, P.; Capasso, F. Science 2015, $347,1342-1345$.

(34) Khorasaninejad, M.; Shi, Z.; Zhu, A. Y.; Chen, W. T.; Sanjeev, V.; Zaidi, A.; Capasso, F. Nano Lett. 2017, 17, 1819-1824.

(35) Faklis, D.; Morris, G. M. Appl. Opt. 1995, 34, 2462-2468.

(36) Sweeney, D. W.; Sommargren, G. E. Appl. Opt. 1995, 34, 24692475.

(37) Stone, T.; George, N. Appl. Opt. 1988, 27, 2960-2971.

(38) Rogers, E. T. F.; Lindberg, J.; Roy, T.; Savo, S.; Chad, J. E.; Dennis, M. R.; Zheludev, N. I. Nat. Mater. 2012, 11, 432-435.

(39) Huang, K.; Liu, H.; Garcia-Vidal, F. J.; Hong, M.; Luk'yanchuk, B.; Teng, J.; Qiu, C.-W. Nat. Commun. 2015, 6, 7059.

(40) Qin, F.; Huang, K.; Wu, J.; Teng, J.; Qiu, C.-W.; Hong, M. Adv. Mater. 2017, 29, 1602721.

(41) Arbabi, A.; Arbabi, E.; Kamali, S. M.; Horie, Y.; Han, S.; Faraon, A. Nat. Commun. 2016, 7, 13682.

(42) Soukoulis, C. M.; Wegener, M. Nat. Photonics 2011, 5, 523530.

(43) Gissibl, T.; Thiele, S.; Herkommer, A.; Giessen, H. Nat. Photonics 2016, 10, 554-560.

(44) Kawata, S.; Sun, H.-B.; Tanaka, T.; Takada, K. Nature 2001, 412, 697-698.

(45) Booth, M.; Wilson, T. J. Microsc. 2000, 200, 68-74. 